\title{
A METROPOLIS ADJUSTED NOSÉ-HOOVER THERMOSTAT
}

\author{
Benedict LeimkuhleR ${ }^{1}$ And Sebastian Reich ${ }^{2}$
}

\begin{abstract}
We present a Monte Carlo technique for sampling from the canonical distribution in molecular dynamics. The method is built upon the Nosé-Hoover constant temperature formulation and the generalized hybrid Monte Carlo method. In contrast to standard hybrid Monte Carlo methods only the thermostat degree of freedom is stochastically resampled during a Monte Carlo step.
\end{abstract}

Mathematics Subject Classification. 65C05, 65C20, 65C60, 82B80, 60H30.

Received October 3rd, 2008.

Published online July 8, 2009.

\section{INTRODUCTION}

Classical molecular dynamics leads naturally to simulations under a constant number of particles, constant volume and constant energy (NVE) ensemble. More often one is however interested in simulations under constant temperature (NVT) or constant pressure and temperature (NPT) conditions. Extended variable formulations are among the most popular avenues to turn an NVE formulation into one that either samples from the corresponding NPT or NVT ensemble [3]. In this paper we focus on the Nosé-Hoover thermostat for constant temperature molecular dynamics simulations $[8,18]$.

The Nosé-Hoover technique is popular because it has been observed in practice to introduce a mild perturbation of dynamics. This means that autocorrelation functions, which measure dynamical relaxation processes, can often be computed with the thermostat active, saving computational effort and reducing complication. Although in widespread use, Nosé dynamics suffers from several shortcomings. First, the method only provides canonical sampling in case the extended system is ergodic; this assumption often fails, e.g. for low-dimensional systems or systems with strong harmonic components. Second, numerical time-stepping errors may lead to a drift in the extended energy, which impacts on the computed ensemble values. The latter point has partially been addressed by the Nosé-Poincaré formulation [4]. However, even when the energy is well controlled, as in the case of Nosé-Poincaré, the distribution will be distorted at large timesteps (but potentially below the stepsize stability threshold) due to numerical errors. Recently, the ergodicity issue has been addressed by the development of stochastic variants of Nosé-Hoover [15,21]. Nevertheless, errors in numerical time-stepping procedures may still adversely affect computed ensemble values.

In this article, we develop a method based on the Nosé-Hoover formulation within the context of Markov chain Monte Carlo (MCMC) techniques [16]. Our approach is based upon an application of the generalized hybrid

\footnotetext{
Keywords and phrases. Molecular dynamics, thermostats, hybrid Monte Carlo, canonical ensemble.

1 The Maxwell Institute and School of Mathematics, University of Edinburgh, Edinburgh, EH9 3JZ, UK. b.leimkuhler@ed.ac.uk

${ }^{2}$ Universität Potsdam, Institut für Mathematik, 14469 Potsdam, Germany.
} 
Monte Carlo method $[1,9,11]$ to Nosé-Hoover dynamics. We demonstrate that it is possible to reduce systematic time-stepping errors by use of a Metropolis condition in combination with a modified rejection procedure, showing the effectiveness of the method in numerical experiments with butane molecules. It is hoped that the relatively weak perturbation used may reduce equilibration issues associated to hybrid Monte Carlo schemes [11] that use a full momenta randomization, for example enhancing the computation of dynamical correlations.

\section{Nosé-Hoover Constant temperature Dynamics}

We consider an $N$-atom molecular system with atomistic positions $\mathbf{r}_{i} \in \mathbb{R}^{3}$, momenta $\mathbf{p}_{i} \in \mathbb{R}^{3}$, and masses $m_{i}, i=1, \ldots, N$, and microcanonical energy function

$$
E=\frac{1}{2} \sum_{i=1}^{N} \frac{\left\|\mathbf{p}_{i}\right\|^{2}}{m_{i}}+V\left(\mathbf{r}_{1}, \mathbf{r}_{2}, \ldots, \mathbf{r}_{N}\right) .
$$

For ease of reference we introduce the notation: $\mathbf{r}=\left(\mathbf{r}_{1}^{T}, \ldots, \mathbf{r}_{N}^{T}\right)^{T}, \mathbf{p}=\left(\mathbf{p}_{1}^{T}, \ldots, \mathbf{p}_{N}^{T}\right)^{T}$, and a diagonal mass matrix $M \in \mathbb{R}^{3 N \times 3 N}$ such that

$$
\mathbf{p}^{T} M^{-1} \mathbf{p}=\sum_{i=1}^{N} m_{i}^{-1}\left\|\mathbf{p}_{i}\right\|^{2}
$$

We consider the Nosé-Hoover equations $[8,18]$

$$
\begin{aligned}
M \dot{\mathbf{r}} & =\mathbf{p} \\
\dot{\mathbf{p}} & =-\nabla_{\mathbf{r}} V(\mathbf{r})-\xi \mathbf{p} \\
\mu \dot{\xi} & =\mathbf{p}^{T} M^{-1} \mathbf{p}-d k_{B} T
\end{aligned}
$$

where $T$ is the temperature, $k_{B}$ is the Boltzmann constant, $\mu>0$ is a constant (the "thermal mass"), and $d$ is the number of degrees of freedom in the system, which we assume for simplicity to be $3 N$. The system $(2.2)-(2.4)$ can be extended by a further variable $\zeta$ :

$$
\dot{\zeta}=d \xi \zeta
$$

in which case the equations possess a first integral ("extended energy")

$$
E_{\mathrm{ENH}}=\frac{1}{2} \mathbf{p}^{T} M^{-1} \mathbf{p}+V(\mathbf{r})+\mu \frac{\xi^{2}}{2}+k_{B} T \ln \zeta .
$$

(We assume $\zeta(0)>0$ so that $\zeta(t)>0$ for all $t>0$.) Assuming ergodicity on a surface of constant extended energy, it can be shown $[12,18]$ that trajectories of the Nosé-Hoover formulation (2.2)-(2.4) sample from an extended distribution of the form

$$
\rho_{\text {ext }}(\mathbf{r}, \mathbf{p}, \xi) \propto \mathrm{e}^{-\beta\left[E+\mu \frac{\xi^{2}}{2}\right]},
$$

where $\beta=\left(k_{B} T\right)^{-1}$. Integrating out with respect to the additional variable, this implies that the phase variables are sampled from the canonical density defined by

$$
\rho_{\text {can }}(\mathbf{r}, \mathbf{p}) \propto \mathrm{e}^{-\beta E} .
$$

Note that the use of (2.5) to define the extended system is slightly different than the original proposal of Nosé [18]. The benefit is that the resulting system of differential equations (2.2)-(2.4) together with (2.5) preserves volume in $(\mathbf{q}, \mathbf{p}, \xi, \zeta)$ space. 
Ergodicity is difficult to establish in practice for the Nosé-Hoover equations (2.2)-(2.4). This problem has given rise to a number of modifications of the basic Nosé-Hoover formulation (see, e.g., [5,14,17]). Most recently, the stochastic Hoover-Langevin method has been proposed [21]:

$$
\begin{aligned}
M \mathrm{~d} \mathbf{r} & =\mathbf{p} \mathrm{d} t \\
\mathrm{~d} \mathbf{p} & =-\nabla_{\mathbf{r}} V(\mathbf{r}) \mathrm{d} t-\xi \mathbf{p} \mathrm{d} t \\
\mu \mathrm{d} \xi & =\mathbf{p}^{T} M^{-1} \mathbf{p} \mathrm{d} t-d k_{B} T \mathrm{~d} t-\mu \gamma \xi \mathrm{d} t+\sqrt{\frac{2 \mu \gamma}{\beta}} \mathrm{d} w
\end{aligned}
$$

where $w(t)$ is a Wiener process [19]. It has been proved rigorously in [15] that (2.7)-(2.9) samples from an extended canonical measure (a measure whose marginal with respect to $\xi$ is (2.6)), in the case of a harmonic potential $V$.

Even when ergodicity can be established for any of the existing Nosé-Hoover formulations, numerical implementations will lead to time series, which, in general, does not sample from the probability density (2.6). The deviation is due to systematic time-stepping errors. These systematic errors can be corrected for by a combination of time-stepping and a probabilistic acceptance/rejection procedure, which rigorously enforces the canonical density (2.6). Such a combination leads to Markov chain Monte Carlo (MCMC) methods [16].

In the remainder of the paper, we develop the MCMC method for the Nosé-Hoover formulation (2.2)-(2.4), which generates a time series $\Gamma_{k}, k=0, \ldots, K$, in the extended state variable

$$
\Gamma_{k}=\left(\mathbf{r}_{k}^{T}, \mathbf{p}_{k}^{T}, \xi_{k}\right)^{T} \in \mathbb{R}^{6 N+1},
$$

which samples from the extended canonical density

$$
\rho_{\mathrm{NH}}(\Gamma) \propto \mathrm{e}^{-\beta E_{\mathrm{NH}}}, \quad E_{\mathrm{NH}}=\frac{1}{2} \mathbf{p}^{T} M^{-1} \mathbf{p}+V(\mathbf{r})+\frac{\mu \xi^{2}}{2} .
$$

\subsection{A Nosé-Hoover timestepping method}

As has been mentioned, the vector field defined by (2.2)-(2.4) together with (2.5) is divergence free and has a first integral $E_{\mathrm{ENH}}{ }^{3}$. For convenience, we abbreviate the system $(2.2)-(2.4),(2.5)$ as

$$
\dot{\mathbf{z}}=f_{\mathrm{ENH}}(\mathbf{z}),
$$

in the phase space variable

$$
\mathbf{z}=\left(\mathbf{r}^{T}, \mathbf{p}^{T}, \xi, \zeta\right)^{T}=\left(\Gamma^{T}, \zeta\right)^{T} \in \mathbb{R}^{6 N+2} .
$$

Observe also that that the equations (2.2)-(2.4), (2.5) are time-reversible under the involution $\mathcal{F}$ defined by

$$
\mathcal{F}:\left(\mathbf{r}^{T}, \mathbf{p}^{T}, \xi, \zeta\right)^{T} \rightarrow\left(\mathbf{r}^{T},-\mathbf{p}^{T},-\xi, \zeta\right)^{T} .
$$

In other words, if $\Phi_{t}$ denotes the time-t-flow map of (2.12), then

$$
\mathcal{F} \Phi_{-t}(\mathcal{F} \mathbf{z})=\Phi_{t}(\mathbf{z})
$$

\footnotetext{
${ }^{3}$ The formulation (2.2)-(2.4), (2.5) is not Hamiltonian; a Hamiltonian formulation for Nosé dynamics with time transformation has been given in [4].
} 
As in [12], we split the equations (2.2)-(2.4), (2.5) into two sets of equations each of which is exactly solvable and each of which is volume conserving and time-reversible. The two systems are:

(A)

$$
\begin{aligned}
M \dot{\mathbf{r}} & =\mathbf{p} \\
\dot{\mathbf{p}} & =0 \\
\mu \dot{\xi} & =\mathbf{p}^{T} M^{-1} \mathbf{p}-d k_{B} T \\
\dot{\zeta} & =0 .
\end{aligned}
$$

(B)

$$
\begin{aligned}
M \dot{\mathbf{r}} & =0, \\
\dot{\mathbf{p}} & =-\nabla_{\mathbf{r}} V(\mathbf{r})-\xi \mathbf{p}, \\
\mu \dot{\xi} & =0, \\
\dot{\zeta} & =d \xi \zeta .
\end{aligned}
$$

Let us denote the associated time-t-flow maps by $\Phi_{t}^{A}$ and $\Phi_{t}^{B}$, respectively. A time-reversible, volume conserving, and second-order numerical method $\Psi_{h}$ is now provided by the composition (Strang splitting)

$$
\Psi_{h}:=\Phi_{h / 2}^{B} \circ \Phi_{h}^{A} \circ \Phi_{h / 2}^{B},
$$

with $h>0$ the step-size.

Let us now make a couple of important observations with regard to the method (2.24). Given a state $\mathbf{z}=\left(\Gamma^{T}, \zeta\right)^{T}$ and a subsequent approximation $\overline{\mathbf{z}}=\left(\bar{\Gamma}^{T}, \bar{\zeta}\right)^{T}=\Psi_{h}(\mathbf{z})$, we find that the difference in extended energy values satisfies

$$
E_{\mathrm{ENH}}(\overline{\mathbf{z}})-E_{\mathrm{ENH}}(\mathbf{z})=E_{\mathrm{NH}}(\bar{\Gamma})-E_{\mathrm{NH}}(\Gamma)+\frac{k_{B} T d \Delta t}{2}(\bar{\xi}+\xi) .
$$

We conclude that this energy difference is independent of the value of $\zeta$. Furthermore, the dynamics in the reduced state variable $\Gamma=\left(\mathbf{r}^{T}, \mathbf{p}^{T}, \xi\right)^{T}$ is also entirely independent of $\zeta$. Hence we view $(2.24)$ as a numerical method in $\Gamma$ only with an energy difference defined by

$$
\Delta E_{\mathrm{ENH}}(\Gamma)=E_{\mathrm{NH}}(\bar{\Gamma})-E_{\mathrm{NH}}(\Gamma)+\frac{k_{B} T d \Delta t}{2}(\bar{\xi}+\xi)
$$

We denote this reduced time-stepping method by $\widehat{\Psi}_{\Delta t}: \Gamma \rightarrow \bar{\Gamma}$. The reduced method $\widehat{\Psi}_{\Delta t}$ shares with $\Psi_{\Delta t}$ the time-reversibility under the involution (2.14). However, while $\Psi_{\Delta t}$ is volume conserving, we obtain

$$
\operatorname{det}\left[D \widehat{\Psi}_{\Delta t}\right](\Gamma)=\exp \left(\frac{d \Delta t}{2}[\bar{\xi}+\xi]\right)
$$

for the reduced time-stepping method. Here $D \widehat{\Psi}_{\Delta t}$ denotes the Jacobian of $\widehat{\Psi}_{\Delta t}$.

\section{Metropolis adjusted Nosé-Hoover (MANH) thermostat}

We now propose a Markov chain Monte Carlo (MCMC) method which rigorously samples from the extended canonical density (2.11). We call this Monte Carlo method the Metropolis adjusted Nosé-Hoover (MANH) thermostat; it is derived as an application of the generalized hybrid Monte Carlo method to the Nosé-Hoover 
equations (2.2)-(2.4). The basic idea is to apply (2.24) to the extended state $\Gamma$ and to accept or reject the outcome based on a Metropolis criterion. Another ingredient of the MANH thermostat is the randomization of the momentum variable $\xi$. This part of the algorithm differs from generalized hybrid Monte Carlo methods where all momenta $\mathbf{p}$ would be randomized. See $[1,9,11]$ for a detailed description of the generalized hybrid Monte Carlo method.

We next provide a detailed description of the MANH algorithm. Given an accepted state $\Gamma_{n}=\left(\mathbf{q}_{n}^{T}, \mathbf{p}_{n}^{T}, \xi_{n}\right)^{T}$, a single MANH step consists of the following two substeps.

\section{Partial thermostat refreshment step}

We randomize the thermostat variable by

$$
\xi_{n}^{*}=\sin (\phi) u+\cos (\phi) \xi_{n}
$$

where $u$ is normally distributed with mean zero and variance $\sigma^{2}=k_{B} T / \mu$ and $\phi \in(0, \pi / 2]$ is a parameter.

\section{Nosé-Hoover Monte Carlo}

(i) Nosé-Hoover dynamics: Given the last accepted and partially refreshed state

$$
\Gamma=\left(\mathbf{q}_{n}^{T}, \mathbf{p}_{n}^{T}, \xi_{n}^{*}\right)^{T},
$$

we augment the state to $\mathbf{z}=\left(\Gamma^{T}, 1\right)^{T}$ and apply the time-reversible and volume-conserving method $(2.24)$ over $M$ time-steps with step-size $h$. We obtain the proposal state

$$
\overline{\mathbf{z}}=\left[\Psi_{h}\right]^{M}(\mathbf{z}) .
$$

(ii) Metropolis criterion: Given a rejection modification operator $\mathcal{A}$, apply the Metropolis acceptance/rejection criterion

$$
\Gamma^{\prime}=\left\{\begin{aligned}
\bar{\Gamma} & \text { with probability } \min (1, \exp (-\beta \delta E)) \\
\mathcal{A} \Gamma & \text { otherwise }
\end{aligned}\right.
$$

with $\bar{\Gamma}$ obtained from $\overline{\mathbf{z}}=\left(\bar{\Gamma}^{T}, \bar{\zeta}\right)^{T}$ and

$$
\delta E:=\sum_{m=0}^{M-1} \Delta E_{\mathrm{ENH}}\left(\left[\Psi_{h}^{m}\right](\Gamma)\right)=E_{\mathrm{ENH}}(\overline{\mathbf{z}})-E_{\mathrm{ENH}}(\mathbf{z}),
$$

with $\Delta E_{\mathrm{ENH}}$ defined by $(2.26)$.

This completes a single MANH step and we set $\Gamma_{k+1}=\Gamma^{\prime}$, where $\Gamma^{\prime}$ is the accepted value (3.4) from the Nosé-Hoover Monte Carlo step.

\subsection{Special Cases}

It can be demonstrated that the method with $\mathcal{A}=\mathcal{F}$ in (31) leads to a MCMC method which satisfies detailed balance. However, numerical experiments presented in the next section confirm that the MANH scheme performs well with $\mathcal{A}=\mathrm{Id}$, which choice would appear to interfere less with the dynamics of the system. We discuss special cases of the MANH thermostat and its relation to existing methods.

(i) If we set $\phi=0$ in (3.1), i.e., $\xi_{k}^{*}=\xi_{k}$, and replace the numerical proposal step (3.3) by the exact propagator in $\Gamma$, i.e., $\bar{\Gamma}=\Phi_{t}\left(\Gamma_{n}\right), t=h M$, then $\Gamma_{k+1}=\bar{\Gamma}$ and MANH reduces to standard Nosé-Hoover dynamics. 
(ii) If we set $M=1$ in (3.3) and $\phi=\sqrt{2 \gamma h} \ll 1$, where $\gamma>0$ is an appropriate constant, then we can Taylor expand (3.1) to obtain

$$
\xi_{k}^{*} \approx(1-\gamma h) \xi_{k}+(2 \gamma h)^{1 / 2} u
$$

We also have that $\bar{\Gamma}$ is an numerical approximation to standard Nosé-Hoover dynamics over a single timestep with initial value $\Gamma$. Hence we may view (3.1) and (3.3) as a composition/splitting method for the stochastic Nosé-Hoover model [15] (2.7)-(2.9). The MANH algorithm combines this discretization with a Metropolis acceptance/rejection criterion (3.4) to insure that we sample exactly from the extended canonical distribution (2.11) even in the presence of numerical time-stepping errors.

(iii) The choice $\phi=\pi / 2$ in (3.1) leads to $\xi_{k}^{*}=u$, i.e., a complete replacement of the thermostat variable $\xi$ after each complete MANH step. This is similar to the standard hybrid Monte Carlo method [6], but note that we do not randomize the complete momentum vector $\mathbf{p}$. The potential benefit is a milder perturbation of the dynamics of the system, which can be expected to reduce equilibration difficulties after randomization.

\subsection{Proof of detailed balance}

The partial thermostat refreshment step (3.1) leads to a normal distribution in $\xi_{n}^{*}$ since $u$ and $\xi_{n}$ are normally distributed with the same variance $\sigma^{2}=k_{B} T / \mu$. Hence we only need to prove that the Nosé-Hoover Monte Carlo step with $\mathcal{A}=\mathcal{F}$ keeps the desired extended canonical density (2.11) invariant.

Recall that the Metropolis criterion (3.4) is equivalent to saying that $\bar{\Gamma}$ is accepted if $r(\bar{\Gamma}, \Gamma) \geq u$, where $u \in[0,1]$ is a uniformly distributed random number and

$$
r(\bar{\Gamma}, \Gamma)=\min \left(1, \frac{\rho_{\mathrm{ENH}}(\overline{\mathbf{z}})}{\rho_{\mathrm{ENH}}(\mathbf{z})}\right), \quad \overline{\mathbf{z}}=\left[\Psi_{\Delta t}\right]^{M}(\mathbf{z}) .
$$

We now set $M=1$ for simplicity and apply (2.26) to obtain

$$
r(\bar{\Gamma}, \Gamma)=\min \left(1, \frac{\rho_{\mathrm{NH}}(\bar{\Gamma})}{\rho_{\mathrm{NH}}(\Gamma)} \mathrm{e}^{\frac{d \Delta t}{2}(\bar{\xi}+\xi)}\right), \quad \bar{\Gamma}=\widehat{\Psi}_{\Delta t}(\Gamma),
$$

which, using (2.27), becomes equivalent to

$$
r(\bar{\Gamma}, \Gamma)=\min \left(1, \frac{\rho_{\mathrm{NH}}(\bar{\Gamma})}{\rho_{\mathrm{NH}}(\Gamma)} \operatorname{det}\left[D \widehat{\Psi}_{\Delta t}\right](\Gamma)\right) .
$$

Let $P(\bar{\Gamma} \mid \Gamma)$ denote the probability density of proposing $\bar{\Gamma}$ in the Nosé-Hoover Monte Carlo step given that the current state is $\Gamma$. The key result of [2] states that a Markov chain Monte Carlo method satisfies a detailed balance relation with respect to the desired invariant distribution $\rho_{\mathrm{NH}}(\Gamma)$ provided that

$$
r(\bar{\Gamma}, \Gamma)=\min \left(1, \frac{\rho_{\mathrm{NH}}(\bar{\Gamma})}{\rho_{\mathrm{NH}}(\Gamma)} \frac{P(\mathcal{F} \Gamma \mid \mathcal{F} \bar{\Gamma})}{P(\bar{\Gamma} \mid \Gamma)}\right),
$$

where $\mathcal{F}$ denotes a linear involution of type (2.14). In case that $P(\bar{\Gamma} \mid \Gamma)$ is induced by a time-reversible and volume conserving time-stepping method, the ratio of $P(\mathcal{F} \Gamma \mid \mathcal{F} \bar{\Gamma})$ and $P(\bar{\Gamma} \mid \Gamma)$ is equal to one and the familiar Metropolis criterion is obtained. In our case, however, the time-stepping method $\widehat{\Psi}_{\Delta t}$ is reversible but not volume conserving and we instead obtain

$$
\frac{P(\mathcal{F} \Gamma \mid \mathcal{F} \bar{\Gamma})}{P(\bar{\Gamma} \mid \Gamma)}=\operatorname{det}\left[D \widehat{\Psi}_{\Delta t}\right](\Gamma),
$$

which implies the desired invariance of $\rho_{\mathrm{NH}}(\Gamma)$ under the MANH thermostat. 
Equation (3.9) can be deduced from the following formal manipulations of the Dirac delta function $\delta$ :

$$
\begin{aligned}
P(\mathcal{F} \Gamma \mid \mathcal{F} \bar{\Gamma}) & =\delta\left(\mathcal{F} \Gamma-\widehat{\Psi}_{\Delta t}(\mathcal{F} \bar{\Gamma})\right) \\
& =\delta\left(\Gamma-\mathcal{F} \widehat{\Psi}_{\Delta t}(\mathcal{F} \bar{\Gamma})\right) \\
& =\delta\left(\Gamma-\widehat{\Psi}_{\Delta t}^{-1}(\bar{\Gamma})\right) \\
& =\delta\left(\widehat{\Psi}_{\Delta t}(\Gamma)-\bar{\Gamma}\right) \operatorname{det}\left[D \widehat{\Psi}_{\Delta t}\right](\Gamma), \\
& =P(\bar{\Gamma} \mid \Gamma) \operatorname{det}\left[D \widehat{\Psi}_{\Delta t}\right](\Gamma) .
\end{aligned}
$$

Here we have used that $\mathcal{F}$ is volume conserving and that $\widehat{\Psi}_{\Delta t}$ is time-reversible under $\mathcal{F}$.

Note that detailed balance does not exclude the possibility of other invariant densities, i.e. the Markov chain may not be irreducible. For a more detailed discussion of irreducibility, the reader is referred to [16].

\section{NUMERICAL EXPERIMENTS}

We implemented the MANH thermostat (with $\phi=\pi / 2)$ in (3.1) and $\mathcal{A}=\operatorname{Id}$ in (3.4) and compared the method with the Nosé-Hoover method $[8,18]$ and the new stochastic dynamics-based Hoover-Langevin scheme [15]. To illustrate the performance of the method, we first simulated a single butane molecule, based on a united atom model as in [20] which treats $\mathrm{CH}_{2}$ and $\mathrm{CH}_{3}$ groups as single bodies. The Hamiltonian is

$$
H=\frac{\left|p_{1}\right|^{2}}{2 m_{1}}+\frac{\left|p_{2}\right|^{2}}{2 m_{2}}+\frac{\left|p_{3}\right|^{2}}{2 m_{3}}+\frac{\left|p_{4}\right|^{2}}{2 m_{4}}+U
$$

where $m_{1,4}=15.03$ atomic mass units, $m_{2,3}=14.03$, and

$$
U=u_{\text {l.b. }}\left(r_{12}\right)+u_{\text {l.b. }}\left(r_{23}\right)+u_{\text {l.b. }}\left(r_{34}\right)+u_{\text {a.b. }}\left(\gamma_{1}\right)+u_{\text {a.b }}\left(\gamma_{2}\right)+u_{\text {d.b. }}(\delta),
$$

with $r_{i j}$ the distance between atoms $i$ and $j, \gamma_{1,2}$ are the cosines of the angles $\angle q_{1} q_{2} q_{3}$ and $\angle q_{2} q_{3} q_{4}$, respectively, and $\delta$ is the cosine of the dihedral formed as the angle between the planes determined by $q_{1}, q_{2}, q_{3}$ and $q_{4}, q_{5}, q_{6}$. The potential terms are

$$
\begin{gathered}
u_{\text {l.b. }}(r)=\frac{K_{1}}{2}\left(r-r_{0}\right)^{2}, \quad u_{\text {a.b. }}(\gamma)=\frac{K_{2}}{2}\left(\gamma-\gamma_{0}\right)^{2}, \quad u_{\text {d.b. }}(\delta)=K_{3}\left(1-4 \delta^{3}+3 \delta\right)+K_{4}(1-\delta), \\
K_{1}=317 \frac{\mathrm{kcal}}{{\mathrm{mol} \AA^{2}}^{2}}, \quad K_{2}=118 \frac{\mathrm{kcal}}{\mathrm{mol}}, \quad K_{3}=1.6 \frac{\mathrm{kcal}}{\mathrm{mol}}, \quad K_{4}=0.6 \frac{\mathrm{kcal}}{\mathrm{mol}} .
\end{gathered}
$$

The interest of course is in more complicated molecules, but a single butane provides an interesting challenge for thermostatting as the length bonds tend to trap energy. We ran our simulation at a cold $k_{B} T=0.1 \mathrm{kcal} \cdot \mathrm{mol}^{-1}$ which made a good challenge for the thermostat. (We also ran the simulation at $k_{B} T=0.4 \mathrm{kcal} \cdot \mathrm{mol}^{-1}$ with qualitatively similar results to those reported below.)

We implemented a standard explicit Nosé-Hoover scheme $[7]^{4}$. The artificial parameter $\mu$ in Nosé-Hoover must be selected to give the best performance of the scheme. We found a broad range of $\mu$ gave similar performance and chose $\mu=5$ (measured relative to AKMA units) within this range. We used stepsizes corresponding to a range of $2-6 \mathrm{fs}$, fixing the time interval at $8 \mathrm{~ns}$, with runs therefore ranging from $1.33 \mathrm{M}$ (million) to

\footnotetext{
${ }^{4}$ To verify correctness we checked the performance of the Nosé-Hoover method against the Nosé-Poincaré method [4] and obtained nearly identical results in this simple example.
} 


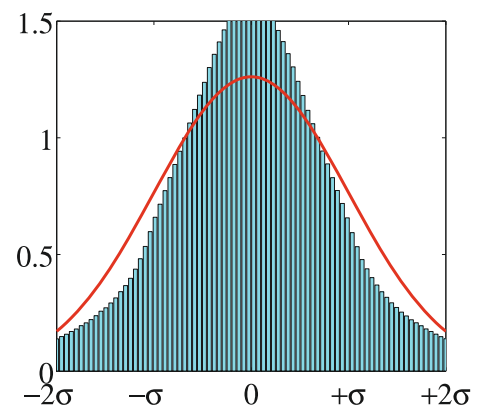

(a) $h=0.006$

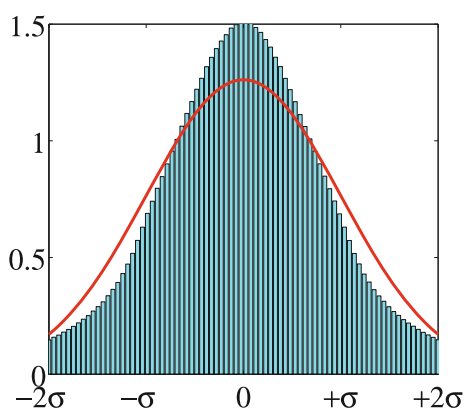

(b) $h=0.004$

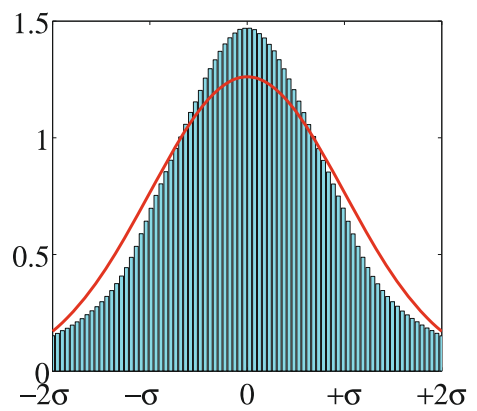

(c) $h=0.002$

Figure 1. Momentum distributions from Nosé-Hoover simulations for three stepsizes.

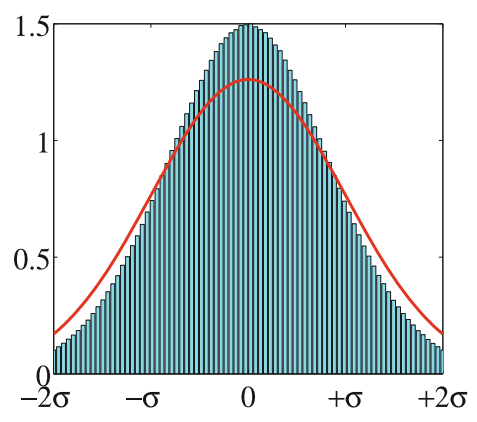

(a) $h=0.006$

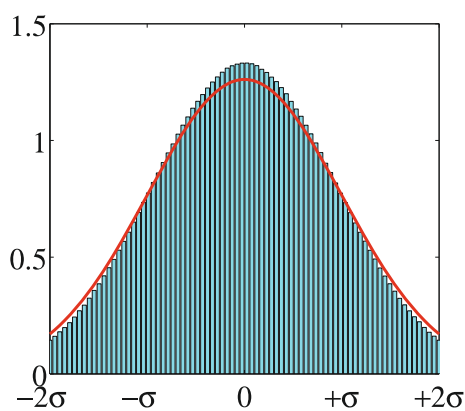

(b) $h=0.004$

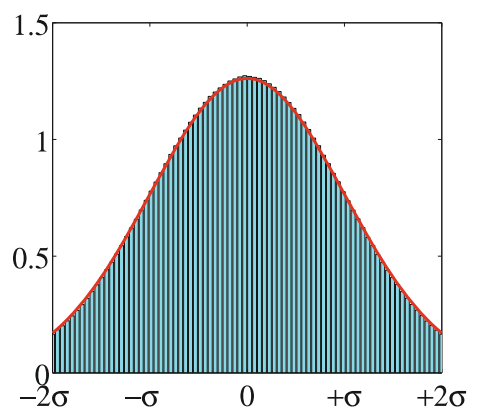

(c) $h=0.002$

Figure 2. Momentum distributions from Hoover-Langevin simulations.

$4 \mathrm{M}$ timesteps. In Figure 1 we show computed histograms of the momenta of all variables, superimposed on a graph of the correct (Gaussian) density for the indicated temperature,

$$
\rho_{\text {mom. }}=\frac{1}{\sqrt{2 \pi m_{i} k_{B} T}} \mathrm{e}^{-\frac{p_{i}^{2}}{2 m_{i} k_{B} T}} .
$$

(This is the density for any of three components of $p_{i}$.) The Nosé dynamics simulation fails to capture the correct canonical sampling properties in this example. The most likely explanation for this failure is that the butane model is close in some sense to a harmonic model; it is well known and even rigorously demonstrated that Nosé dynamics is not ergodic for such systems [13].

We next used the recently proposed Hoover-Langevin thermostat (2.7)-(2.9). Again the parameter $\mu$ must be selected to obtain a suitable coupling of bath to physical variables, and we used the same value $\mu=5$ used for the previous experiments. The additional parameter $\gamma$ in the Hoover-Langevin method regulates the strength of the noise term which is introduced. We chose $\gamma=5 \mathrm{ps}^{-1}$. (Smaller values gave relatively poorer performance.)

The performance is much better (see Fig. 2). The Hoover-Langevin has been rigorously analysed in [15] for a harmonic model, and it is likely that the strong harmonic bond is the dominant challenge here, so this experiment agrees with theory. At larger stepsizes, the distribution becomes distorted due to numerical error. The addition of noise has corrected the ergodicity problem in the Nosé-Hoover thermostat but has not corrected the distribution for the effects of discretization error.

Finally we tested the MANH thermostat against the same model problem. The relevant parameters here are $\mu$ regulating the coupling of the artificial and physical variables, and $M$, the number of steps taken between 


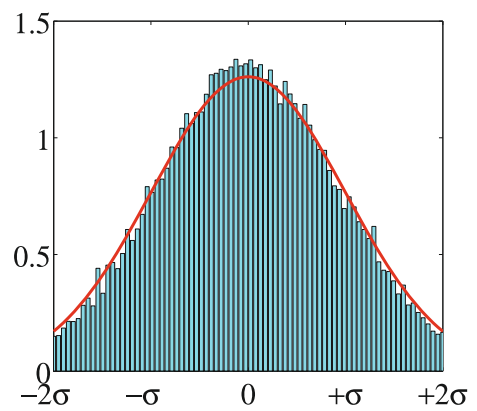

(a) $h=6$ fs

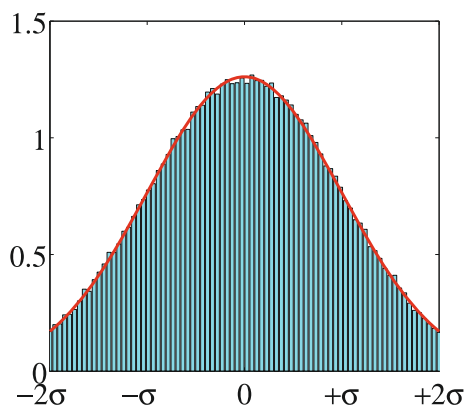

(b) $h=4 \mathrm{fs}$

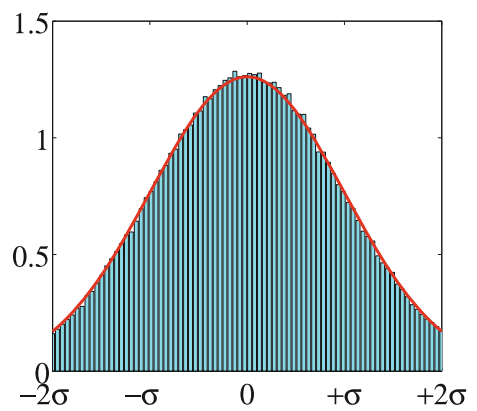

(c) $h=2$ fs

Figure 3. Momentum distributions from MANH simulations.

resampling of the thermostat degree of freedom $\xi$. We chose $\mu=1$ and $M=100$. The results corresponding to the previous experiments are shown in Figure 3. Clearly the MANH simulations provide a more accurate momentum distribution in this example. Note that the slightly rough appearance of the MANH histograms is due to the fact that much less data is available from a MCMC type simulation involving a similar number of steps to a standard method involving the same number of basic steps; these are further improved in longer simulations.

Errors in temperature are shown in Figure 4. It is interesting to note that even when Nosé-Hoover is completely inaccurate in terms of the distribution, as here, the accuracy of the average kinetic energy compared to the target temperature may be quite high. This is attributable to the explicit kinetic energy control law that forms part of Nosé-Hoover. Both the Nosé-Hoover and Hoover-Langevin methods show a quadratic dependence on stepsize, as is to be expected since the error in temperature is related to numerical discretization error. MANH on the other hand, shows an erratic fluctuation of average kinetic energy error, since it is dominated by sampling error in this case, i.e., the number of MC samples is smaller for the experiments with larger step-sizes $h$.

To examine the asymptotic behavior of MANH, we show in Figure 5 below the convergence of average kinetic energy to temperature in a simulation of roughly 140000 timesteps, with here, $h=6$ fs.

One might question whether the convergence of temperature or recovery of the kinetic energy distribution is evidence proper canonical sampling. For this purpose we have computed the distribution of the dihedral angle in Butane-1 via a histogram after various numbers of steps of MANH. Parameters here were $k_{B} T=$ $0.594 \mathrm{kcal} \cdot \mathrm{mol}^{-1}$ corresponding to $300 \mathrm{~K}$, and we set $h=1 \mathrm{fs}, \mu=1$, and took 50 steps before Metropolis test. The exact density of the dihedral angle is easily computed for Butane-1; it is just

$$
\varphi(\psi) \propto \mathrm{e}^{-\beta u_{\mathrm{d} . \mathrm{b}}}
$$

where $\psi$ is the dihedral angle and $u_{\text {d.b. }}$ is the dihedral bending energy [10]. In Figure 6 , the convergence of the dihedral distribution to its nominal form is illustrated with simulations of $2 M, 8 M$ and $32 M$ steps. Relatively large numbers of timesteps are needed here for accurate statistics because, even at this temperature (corresponding to $300 \mathrm{~K}$ ), transitions between the different butane conformations are relatively rare.

\subsection{Simulation of Liquid Butane-8}

Although we have not conducted exhaustive experiments with larger systems, we did examine a system of 8 butane molecules in periodic boundary conditions. In this model a Lennard-Jones interaction is introduced 


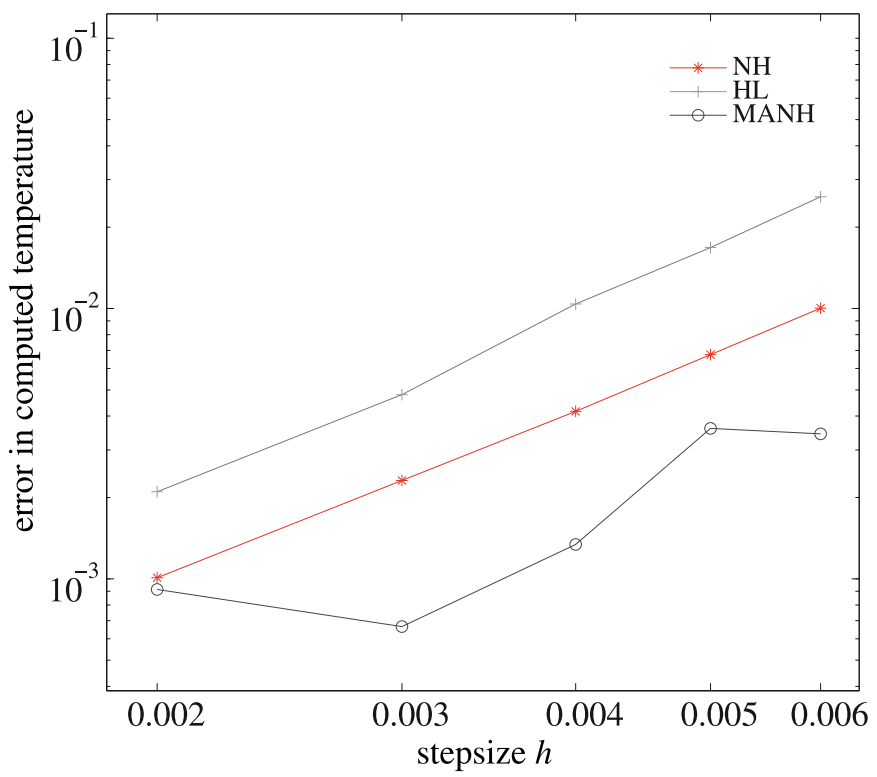

FIgURE 4. Discrepancy of average kinetic energy from temperature for the three methods.

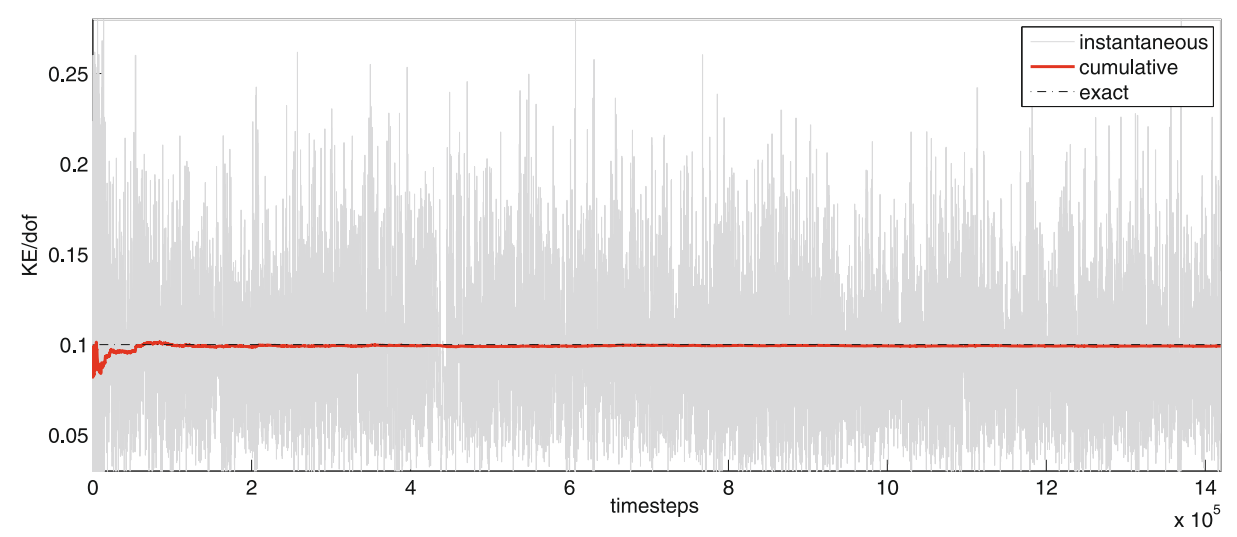

FiguRE 5. Convergence of average kinetic energy to temperature in a longer MANH simulation.

between all pairs of atoms of different molecules, of the form

$$
\phi_{\mathrm{LJ}}(r)=4 \epsilon\left[\left(\frac{\sigma}{r}\right)^{12}-\left(\frac{\sigma}{r}\right)^{6}\right] .
$$

The coefficients used were $\epsilon=0.14307 \mathrm{kcal} \cdot \mathrm{mol}^{-1}$ and $\sigma=3.923 \AA$. A temperature of $k_{B} T=0.4 \mathrm{kcal} \cdot \mathrm{mol}^{-1}$ was used in these simulations, corresponding to a liquid state simulation (at around $240 \mathrm{~K}$ ). We again compared the Nosé-Hoover, Hoover-Langevin and MANH algorithms. The only difference in parameters from the butane- 1 simulation is that we have here used $\mu=3$, which gave good results in the limit of small stepsize using all three methods, and we took 50 instead of 100 steps between Metropolis randomizations in MANH, which improved the convergence behavior. In this application, the first two methods performed very similarly, for all choices of stepsize corresponding to a range of 2 fs to $8 \mathrm{fs}$, the latter being the stability threshold. This indicates 

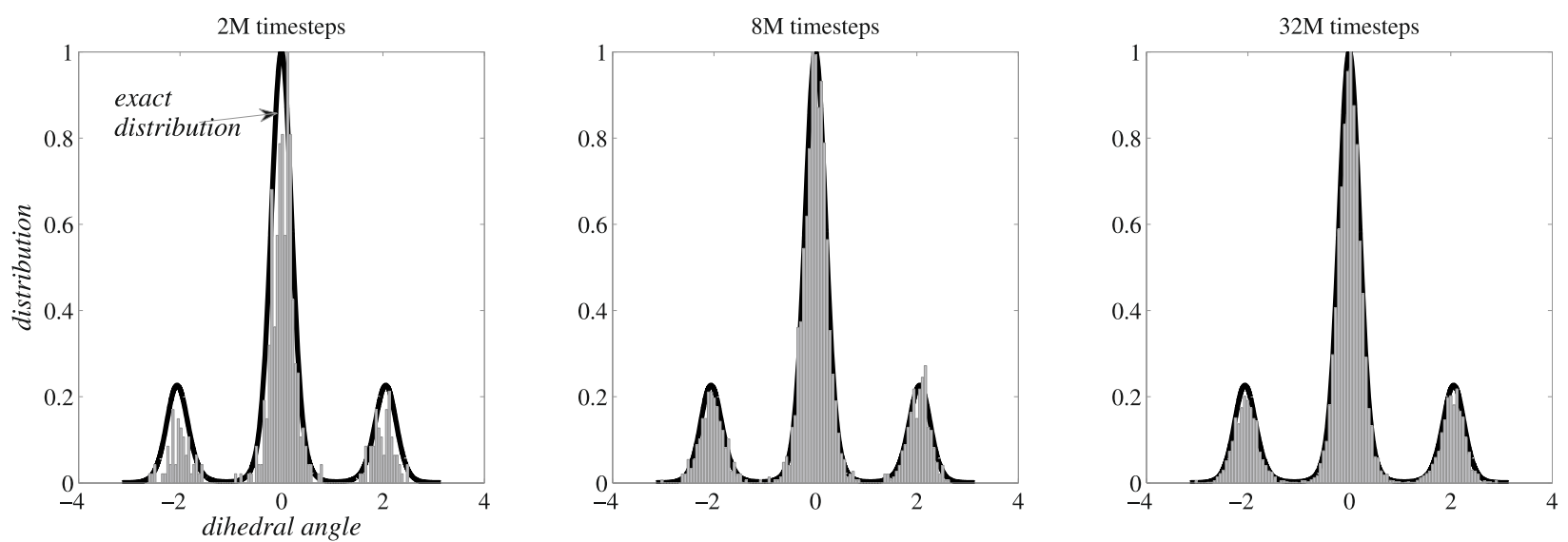

FigurE 6. Convergence of the dihedral angle distribution.
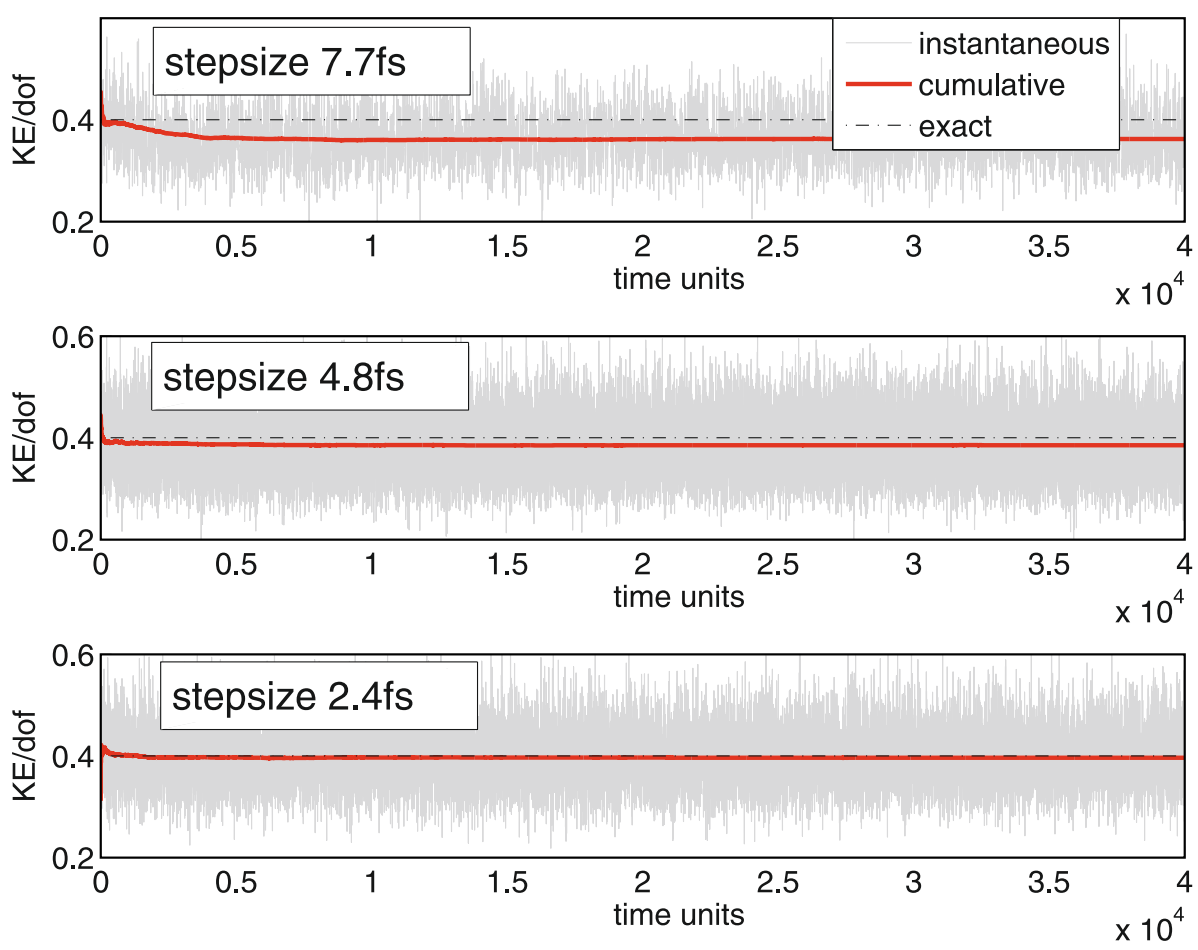

Figure 7. Butane-8 simulations. Kinetic energy per degree of freedom (cumulative and instantaneous) graphed against time, for each of three stepsizes using the Hoover-Langevin method. Nosé-Hoover results were similar. Note the persistent gap between asymptotic cumulative average and target temperature visible at the larger stepsizes.

that the molecular problem is likely ergodic so the additional stochastic perturbation of the Hoover-Langevin method is not strictly necessary for this purpose (although both methods have a stepsize-related defect). Moreover, the temperature convergence provides a diagnostic measure for the overall performance, so we report here 

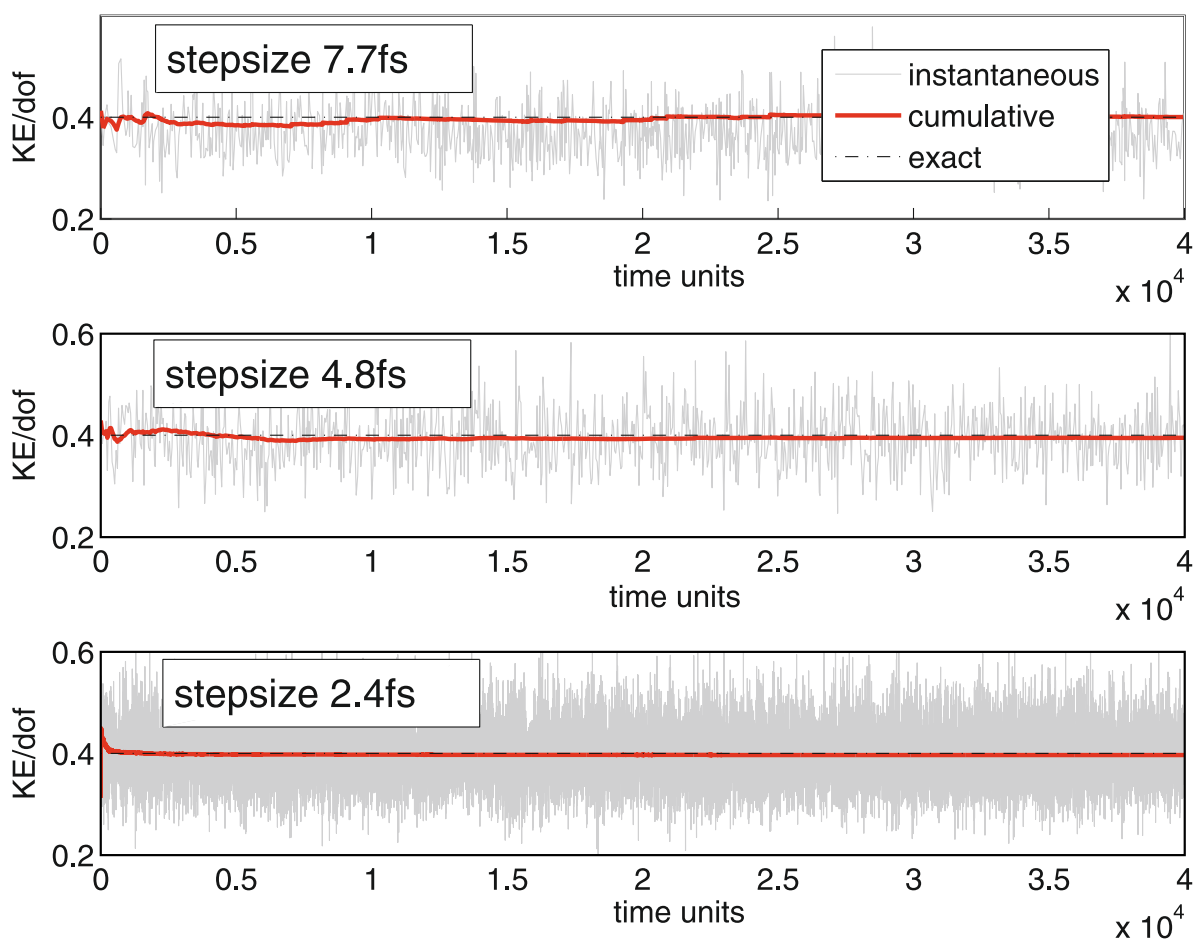

Figure 8. Butane-8 simulations. Kinetic energy per degree of freedom (cumulative and instantaneous) graphed against time, for each of three stepsizes using the MANH thermostat. Note the convergence to target temperature.

only the temperature convergence, and only for the Hoover-Langevin and MANH methods. The convergence results for average kinetic energy to temperature are summarized in Figures 7 (Hoover-Langevin) and 8 (MANH), for various values of the stepsize. It is clear that the differences in performance are qualitatively similar to what was reported for butane-1.

\section{Open PROBLEM}

Our motivation for using the Nosé-Hoover method is that noise can be introduced in a secondary fashion into the dynamical equations, with potential benefits for the recovery of averaged dynamics (autocorrelation functions and transport coefficients). In this article we have not, however, directly addressed the question of the magnitude of this perturbation which is likely to problem specific. The assessment of the influence of the stochastic resampling on correlation functions is an open challenge.

\section{REFERENCES}

[1] E. Akhmatskaya and S. Reich, GSHMC: An efficient method for molecular simulations. J. Comput. Phys. 227 (2008) $4934-4954$.

[2] E. Akhmatskaya, N. Bou-Rabee and S. Reich, Generalized hybrid Monte Carlo methods with and without momentum flip. J. Comput. Phys. 227 (2008) 4934-4954.

[3] M.P. Allen and D.J. Tildesley, Computer Simulation of Liquids. Clarendon Press, Oxford (1987)

[4] S.D. Bond, B.J. Leimkuhler and B.B. Laird, The Nosé-Poincaré method for constant temperature molecular dynamics. J. Comput. Phys. 151 (1999) 114-134.

[5] G. Bussi, D. Donadio and M. Parrinello, Canonical sampling through velocity rescaling. J. Chem. Phys. 126 (2007) 014101.

[6] S. Duane, A.D. Kennedy, B.J. Pendleton and D. Roweth, Hybrid Monte-Carlo. Phys. Lett. B 195 (1987) $216-222$.

[7] D. Frenkel and B. Smit, Understanding Molecular Simulation. Academic Press, New York (1996). 
[8] W.G. Hoover, Canonical dynamics: Equilibrium phase-space distributions. Phys. Rev. A 31 (1985) $1695-1697$.

[9] A.M. Horowitz, A generalized guided Monte-Carlo algorithm. Phys. Lett. B 268 (1991) $247-252$.

[10] J.A. Izaguirre and S.S. Hampton, Shadow Hybrid Monte Carlo: An efficient propagator in phase space of macromolecules. J. Comput. Phys. 200 (2004) 581-604.

[11] A.D. Kennedy and B. Pendleton, Cost of the generalized hybrid Monte Carlo algorithm for free field theory. Nucl. Phys. B 607 (2001) 456-510.

[12] P. Klein, Pressure and temperature control in molecular dynamics simulations: a unitary approach in discrete time. Modelling Simul. Mater. Sci. Eng. 6 (1998) 405-421.

[13] F. Legoll, M. Luskin and R. Moeckel, Non-ergodicity of the Nose-Hoover thermostatted harmonic oscillator. Arch. Ration. Mech. Anal. 184 (2007) 449-463.

[14] B. Leimkuhler and C. Sweet, A Hamiltonian formulation for recursive multiple thermostats in a common timescale. SIAM J. Appl. Dyn. Syst. 4 (2005) 187-216.

[15] B. Leimkuhler, E. Noorizadeh and F. Theil, A gentle ergodic thermostat for molecular dynamics. J. Stat. Phys. (2009), doi: 10.1007/s10955-009-9734-0.

[16] J.S. Liu, Monte Carlo Strategies in Scientific Computing. Springer-Verlag, New York (2001).

[17] G.J. Martyna, M.L. Klein and M. Tuckerman, Nose-Hoover chains: The canonical ensemble via continuous dynamics. J. Chem. Phys. 97 (1992) 2635-2643.

[18] S. Nosé, A unified formulation of the constant temperature molecular-dynamics methods. J. Chem. Phys. 81 (1984) 511-519.

[19] B. Oksendal, Stochastic Differential Equations. 5th Edition, Springer-Verlag, Berlin-Heidelberg (2000).

[20] J.-P. Ryckaert and A. Bellemans, Molecular dynamics of liquid alkanes. Faraday Discussions 66 (1978) 95-107.

[21] A. Samoletov, M.A.J. Chaplain and C.P. Dettmann, Thermostats for "slow" configurational modes. J. Stat. Phys. 128 (2007) $1321-1336$ 